\title{
PFLEGE PERSPEKTIVEN
}

Freiwillige helfen jetzt Das BMFSFJ-Online-Angebot www.freiwillige-helfen-jetzt.de ist freigeschaltet. Die Plattform will lokale und regionale Kontakte erleichtern zwischen Freiwilligendienstleistenden aus BFD, FSJ und FÖJ (Bundesfreiwilligendienst, Freiwilligem Sozialen Jahr und Freiwilligem Ökologischen Jahr), die im Moment nicht an ihren eigentlichen Einsatzorten tätig sein können. + +++ Personalie an der PTHV Die Pflegewissenschaftlerin Prof. Dr. Brigitte Anderl-Doliwa wurde zur Honorarprofessorin an der Philosophisch-Theologischen Hochschule Vallendar (PTHV) ernannt. +++ Betriebliches Gesundheitsmanagement Im Zuge der Krise greifen Unternehmen verstärkt auf digitale Instrumente zurück: Homeoffice, Webkonferenzen, Digital Learning, digitale Beratung und Betreuung. Dies kann bei allen Chancen die Mitarbeiter auch belasten. Mit der Initiative BGM 4.0 wollen die Betriebskrankenkassen gegensteuern. +++ Programm zur Selbsthilfe Der Bundesverband privater Anbieter sozialer Dienste e.V. (bpa) hat ein Selbsthilfeprogramm zur Beschaffung von Schutzausrüstung aufgelegt und dafür neun Millionen Euro zur Vorfinanzierung bereitgestellt.

Weitere Meldungen: $\square$ springerpflege.de

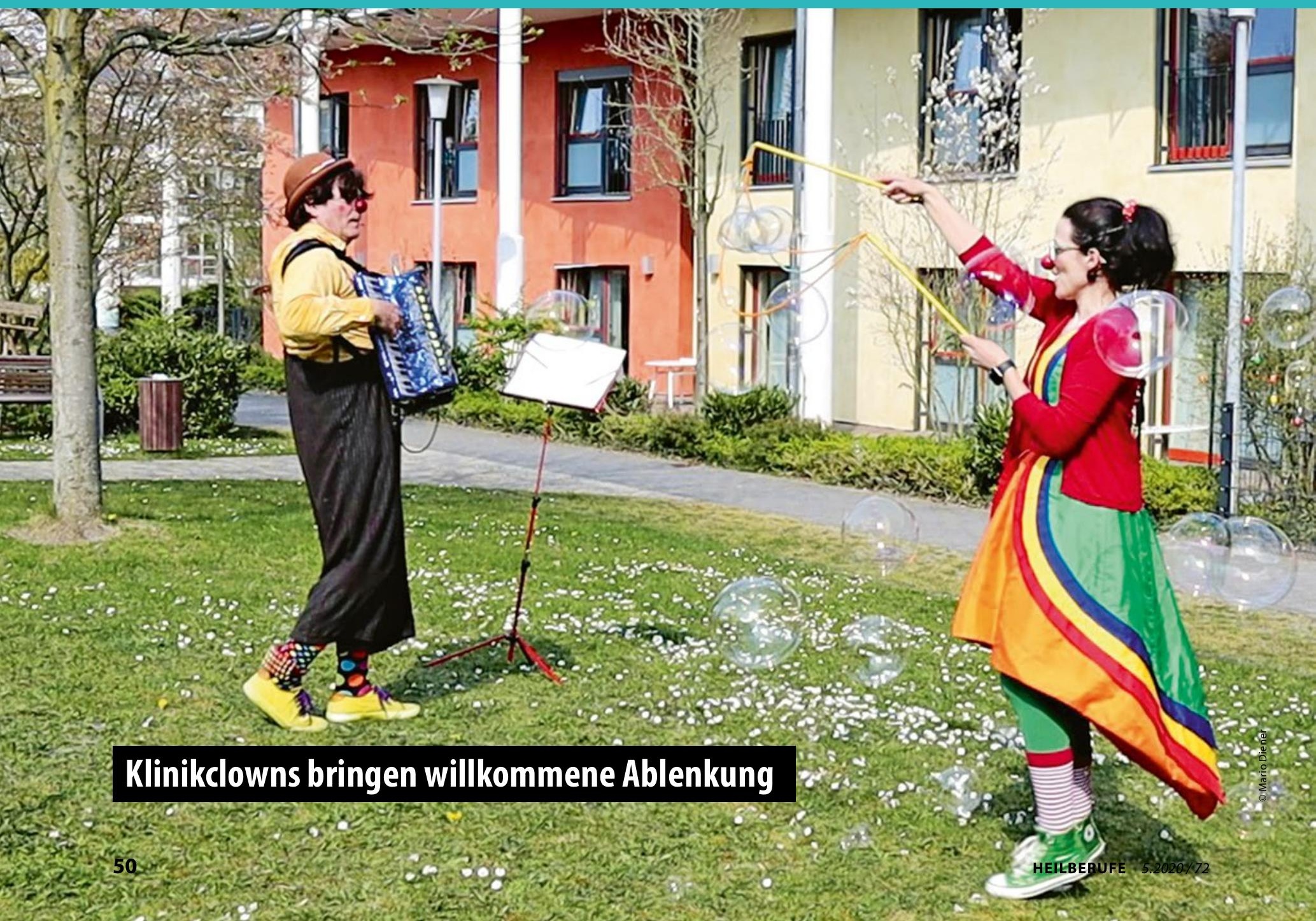




\subsection{Freiwillige haben eine \\ Einsatzstelle in Gesundheit und Pflege.}

\section{BESUCHSVERBOT IN HEIMEN \\ Kreativität gefragt: Klinikclowns zu Gast}

Besuchsverbote belasten in Krankenhäusern und Pflegeeinrichtungen die ohnehin oft schwere Situation für kranke und pflegebedürftige Menschen noch zusätzlich, oft kommt Ablenkung und persönliche Fürsorge zu kurz, Einsamkeit und Ängste machen sich breit. Die Klinikclowns in den Mitgliedsvereinen des Dachverbands Clowns in Medizin und Pflege Deutschland e.V. halten dennoch und gerade jetzt die Verbindung zu Bewohnern von Pflegeheimen und Kindern und erwachsenen Patienten im Krankenhaus und stehen ihnen mit Humor und Zuversicht in dieser schweren Zeit zur Seite. „Mit Online-Live-Visiten bleiben die Klinikclowns in Kontakt mit kleinen Patienten und ihren Angehörigen, mit Postkarten, Briefen und Geschenkpäckchen halten sie die Verbindung zu Senior*innen in Pflegeheimen und zu den Mitarbeiter*innen in den Einrichtungen, die zur Zeit so vieles leisten müssen", erklärt die Vorsitzende des Dachverbands Elisabeth Makepeace. Und, wo das möglich ist, besuchen die Clowns die Vorplätze oder Innenhöfe verschiedener Einrichtungen, so dass die Patienten und Altenheimbewohner vom Fenster aus dabei sein können, wie zum Beispiel kürzlich in Idstein.

dachverband-clowns.de

\section{QUALITÄT UND SICHERHEIT}

\section{Pflege-Azubis schützen}

Die neue Pflegeausbildung ist zum 1. April gestartet. Angesichts der Corona-Epidemie fürchtet der Deutsche Berufsverband für Pflegeberufe (DBfK) eine Überforderung von Azubis und warnt davor, sie unvorbereitet in Praxiseinsätze zu schicken. „Der Ausbildungsgrundsatz, dass Lernende nur mit Aufgaben betraut werden dürfen, die ihrem Ausbil- dungsstand entsprechen und die der Erreichung des Ausbildungszieles dienen, gilt in der gegenwärtigen Situation ganz besonders", erklärte DBfK-Präsidentin Christel Bienstein. Es wäre die "schlechteste Lösung ", wenn neue Auszubildende und Studierende unvorbereitet in die Pflege-Praxis geschickt würden. Der DBfK sieht die Gefahr von Ausbildungsabbrüchen bis hin zum Ausstieg aus der Ausbildung, „bevor sie begonnen hat". Der Verband fordert daher, neue Auszubildende nicht ohne vorbereitenden Unterricht in der Praxis einzusetzen. Zudem müssten die Schulen am Praxisort auch in der Pandemie eine geeignete Begleitung gewährleisten. Nicht zuletzt sollten Lernende bevorzugt dort eingesetzt werden, wo sie weniger COVID-19-Erkrankte antreffen. "Lernende dürfen nur dann in der Praxis eingesetzt werden, wenn die Praxisanleitung sichergestellt und eine Überforderung sicher verhindert werden kann", so Bienstein.

Franz Wagner, Präsident des Deutschen Pflegerats (DPR), forderte darüber hinaus, dass die Pflegeschulen stärker in die Umsetzung von digitalen Infrastrukturen, E-LearningAngeboten und in die dafür notwendige Ausstattung investieren. Diese Investitionsmaßnahmen müssten durch die Länder unterstützt werden, Mittel aus den Länderhaushalten und dem Digitalpakt des Bundes schnell und unkompliziert ohne lange Antragsverfahren von den Schulen abgerufen werden können.

$\square$ dbfk.de

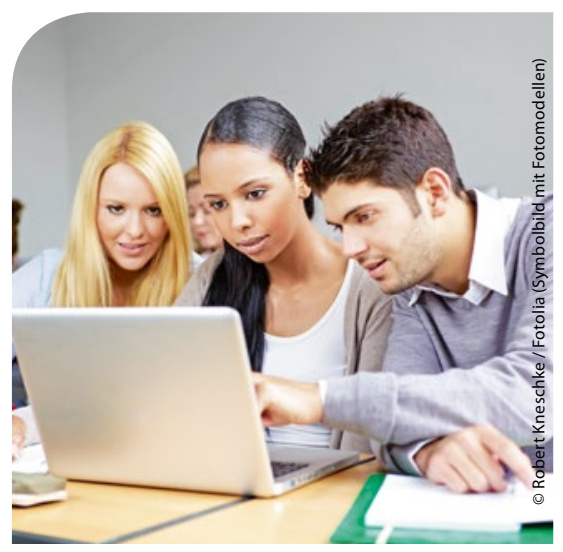

\section{RHEINLAND-PFALZ}

\section{Freiwilligen-Pool stößt auf Zustimmung}

Die Landespflegekammer Rheinland-Pfalz verbuchte am 14. April bereits 450 Meldungen an den Freiwilligen-Pool. Die Kammer hat in Zusammenarbeit mit dem Land eine zentrale Meldestelle für Pflegefachpersonen und Pflegehilfskräfte eingerichtet, die für einen befristeten Zeitraum wieder in den Pflegeberuf zurückkehren möchten, um bei der Bewältigung der Corona-Pandemie zu unterstützen.

pflegekammer-rlp.de

\section{PFLEGEFORSCHUNG}

\section{DIP zieht Bilanz}

Das Deutsche Institut für angewandte Pflegeforschung e.V. (DIP) mit Sitz in Köln hat den Geschäftsbericht für den Zeitraum der Jahre 2016 bis 2018 vorgelegt. Im Berichtszeitraum wurden insgesamt 31 Projekte im Gesamtvolumen von mehr als 2,8 Mio. Euro bearbeitet. Dies bedeutet eine Steigerung der Erträge und Aufwendungen zum Vorberichtszeitraum um rund ein Drittel. Aus der Projektarbeit sind u.a.. rund 60 Publikationen entstanden. Viele Berichte lassen sich kostenlos von der Institutshomepage herunterladen. „Das DIP hat in den vergangenen Jahren wieder eine ganze Reihe wichtiger Themen und Vorhaben zur Innovation der Pflege angepackt und daraus viele Ergebnisse der Öffentlichkeit zur Verfügung gestellt. Ich freue mich, dass das Institut damit wiederum seinem Satzungsauftrag als gemeinnützige Forschungseinrichtung eindrucksvoll nachkommen konnte", sagte Prof. Dr. Frank Weidner, Vorstandsvorsitzender des DIP. Wichtige Themenfelder waren und sind u.a. die Entwicklung und Umsetzung des Pflegeberufegesetzes, Pflegeprävention, neue Technologien und Digitalisierung in der Pflege.

dip.de 
Frühe Hilfen - das ist das Thema in der Mai-Ausgabe der PflegeZeitschrift. Jetzt Einzelheft bestellen.

\section{CORONA-PANDEMIE}

\section{Kostenloses Buchpaket für Fortbildung}

Deutsche Kliniken müssen derzeit - im Falle eines plötzlichen Anstiegs von Schwerst-Infizierten - in kürzester Zeit mehr Fachpersonal für die Anforderungen auf Intensivstationen zur Verfügung haben. Aufgrund des häufig vorhandenen Personalmangels werden auch Mitarbeiter von anderen Stationen herangezogen, denen das spezielle Fachwissen aus der Intensivmedizin und der Pneumologie fehlt. Dies kann bedeuten, dass bereits früher Gelerntes wieder aufgefrischt oder neues Wissen aus den geforderten Spezialgebieten Pneumologie und Intensivmedizin, vermittelt werden muss. Auf jeden Fall heißt es: schnell einen qualifizierten Wissensstand zu erwerben und anwenden zu können.

Der Wissenschaftsverlag Springer Nature schaltet über seine Plattform SpringerLink (link.springer.com) ein Buchpaket mit deutschsprachigen Lehr- und Fachbüchern aus den Gebieten Intensivmedizin und Pneumologie frei. Die ausgewählten Titel wenden sich an pflegerisches und medizinisches Fachpersonal. Dieses Paket ist ein kostenloses Angebot für klinische Einrichtungen in der aktuellen medizinischen Ausnahmesituation: Der Wis-

\section{@Pringer Plege}

Kostenloses Buchpaket: Intensivmedizinische Betreuung von Corona-Patienten

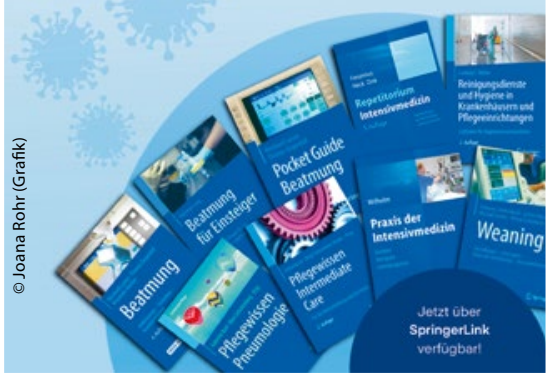

senschaftsverlag möchte Krankenhäusern dabei helfen, sein Personal so fortzubilden, dass es ausreichend qualifiziert ist, die vermehrt erwarteten Corona-Patienten mit schwerem Verlauf kompetent zu versorgen.

link.springer.com

\section{BUNDESKABINETT}

\section{Beschlossen: Gesetz zum Patientendaten-Schutz}

Digitale Lösungen schnell zum Patienten bringen und dabei sensible Gesundheitsdaten bestmöglich schützen - das ist das Ziel des Entwurfs eines „Patientendaten-Schutz-Gesetzes". Das Bundeskabinett hat den Entwurf Anfang April beschlossen. Geregelt ist: Krankenkassen müssen ihren Versicherten ab 2021 eine elektronischen Patientenakte anbieten. Damit diese auch befüllt wird, erhalten Patienten zeitgleich einen Anspruch darauf, dass ihr Arzt Daten in die ePA einträgt. Ärzte und Krankenhäuser, die die ePA erstmals befüllen, bekommen 10 Euro. Für die Unterstützung der Versicherten bei der weiteren Verwaltung ihrer ePA erhalten Ärzte, Zahnärzte und Apotheker ebenfalls eine Vergütung. Die Nutzung der elektronischen Patientenakte ist freiwillig. Der Versicherte entscheidet, welche Daten in der ePA gespeichert oder wieder gelöscht werden. Er entscheidet auch in jedem Einzelfall, wer auf die ePA zugreifen darf. Neben Befunden, Arztberichten oder Röntgenbildern lassen sich ab 2022 der Impfausweis, der Mutterpass, das gelbe U-Heft für Kinder und das Zahn-Bonusheft in der elektronischen Patientenakte speichern. Versicherte können ab 2022 bei einem Krankenkassenwechsel ihre Daten aus der ePA übertragen lassen.

Das Gesetz soll voraussichtlich im Herbst in Kraft treten. Es ist im Bundesrat nicht zustimmungspflichtig.

bundesgesundheitsministerium.de

\section{URTEIL \\ Aus für Antrag gegen Pflegeheim-Erweiterung}

Auch wenn kranke und behinderte Menschen manchmal ungewöhnliche Laute von sich geben, können solche „Lebensäußerungen“ niemals eine "schädliche Umwelteinwirkung" sein. Das hat das Oberverwaltungsgericht (OVG) Nordrhein-Westfalen in Münster betont (Az.: 10 B 312/20).

Es wies damit einen Eilantrag von Anwohnern gegen die Baugenehmigung für die Erweiterung eines Pflegeheims in Essen-Heidhausen ab. Diese machten geltend, sie hätten durch das Heim schon jetzt eine „enorme Geräuschkulisse" zu verkraften. Mit der Erweiterung rückten diese Geräusche noch näher an ihr Grundstück heran. Das OVG Münster zeigte für diese Sorgen wenig Verständnis. Ein Pflegeheim gelte baurechtlich als Wohnnutzung. Dabei verstehe es sich von selbst, "dass die Lautäußerungen von kranken oder behinderten Bewohnern, auch wenn sie auf einem benachbarten Grundstück deutlich wahrgenommen werden könnten, keine schädlichen Umwelteinwirkungen" seien. Deshalb könnten sie auch nicht zu einem Verstoß gegen das baurechtliche Rücksichtnahmeverbot führen.

- Stadt kam Anwohnern entgegen: Hier war die Stadt Essen den Anwohnern sogar noch mit den der Baugenehmigung beigefügten Nebenbestimmungen entgegengekommen. So wurde der Heimbetreiber verpflichtet, bei „außergewöhnlichen Lärmereignissen" in den den Nachbarn zugewandten Zimmern die Fenster zu schließen. Solche Auflagen seien gar nicht nötig gewesen, so das OVG. Ob sie ausreichen und konkret genug sind, spiele daher keine Rolle.

Auch den Lärm von Rettungswagen und nach ausgebüchsten Bewohnern suchenden Hubschraubern müssten die Anwohner hinnehmen. Es handele sich dabei um „sozialadäquate Auswirkungen von Maßnahmen zur Gefahrenabwehr beziehungsweise zur Rettung von Personen".

(mwo) 


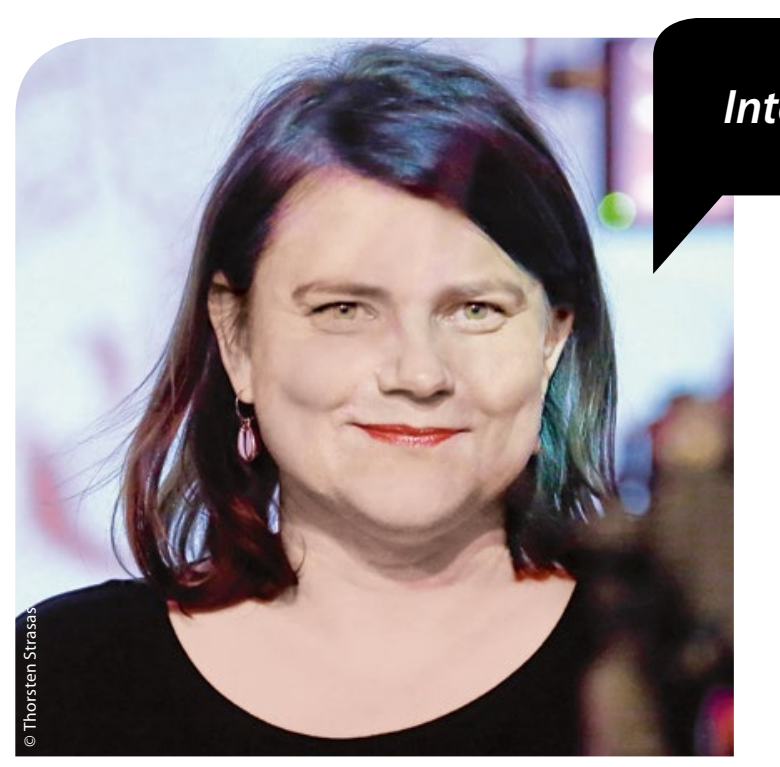

... mit Yvonne Falckner. Die Initiatorin des "CareSlam" arbeitet als Pflegefachkraft in Berlin. Zusammen mit Kollegen hat sie eine Online-Petition an Bundesgesundheitsminister Jens Spahn gestartet.

_ Frau Falckner, seit die Corona-Pandemie auch Deutschland erreicht hat, weiß plötzlich jeder: Pflegefachkräfte sind systemrelevant. Wie fühlt sich diese "Adelung"Ihres Berufs an?

Falckner: Das fühlt sich schon etwas überfordernd an. Es ist nicht genau erkennbar: Handelt es sich um einen Rückzug auf einfache Symbolhandlungen oder geht die Anerkennung letztlich mit einem echten Perspektivwechsel einher? Ein anderer Blick auf die Pflege und ihre Bedeutung für die Gesellschaft wäre so wichtig.

Wenn das Klatschen nur eine leere Handlung darstellt, steht die Pflege nach Corona wieder nur mit leeren Händen da. Pflege wird weiter vor sich hinplätschern, bis die Quelle schließlich versiegt. Und wenn man sich die Entwicklung der Pflege anschaut, könnte das schneller geschehen als erwartet: Fachkräftemangel, sinkende Ausbildungszahlen, eine kurze Verweildauer im Beruf und in den nächsten Jahren erreichen viele Fachkräfte das Rentenalter. Wir Pflegefachkräfte sollten endlich als ein wichtiges Fundament der Gesellschaft verstanden werden.

_ Personalmangel und Dauerüberlastung - Pflegefachkräfte arbeiten seit langem am Limit. Was macht die aktuelle Situation denn besonders deutlich?

Falckner: Corona zeigt, dass wir eine akute Krise auf einer chronischen Krise haben. Das ist immer eine schlechte Kombination. Die Ressourcen waren schon vorher unzureichend. Nun müssen wir unter solchen Voraussetzungen eine akute Gefahr abwehren.

Pflege hat einen sehr hohen Stellenwert, auch wenn es darum geht, andere Systeme, das gesellschaftliche Leben zu stützen. Das ist aber nur möglich, wenn wir mit ausreichend Schutzmaterial ausgestattet werden. Wir haben nichts davon, wenn Pflege zum Dauerspreader (= Dauerverbreiter des Virus) wird und das öffentliche Leben auf lange Zeit eingeschränkt werden muss. Es muss allen klar sein, dass wir gemeinsam in dieser schwierigen Situation die Daseinsvorsorge gewährleisten.
Beruf den Rücken gekehrt haben, zurückgewinnen. Das wird nur mit einer ernstgemeinten Einladung unter Akzeptanz der Wahlfreiheit und Mündigkeit des Einzelnen klappen. Pflege ist nicht die Spielzeugkiste des Gesundheitssystems, die man mit Herzlichkeitsfloskeln locken kann. Es ist auch nicht zu verstehen, weshalb ein Facharbeiter, der in der Industrie am Band arbeitet, besser bezahlt wird

\section{- Pflege darf nach Corona nicht mit leeren Händen dastehen.}

_ Immerhin wurden die Beatmungskapazitäten in Krankenhäusern ausgebaut ... Falckner: Ohne genügend Fachkräfte, die sie bedienen, haben auch zusätzliche Beatmungsgeräte nur einen homöopathischen Effekt. Es braucht auch Fachkräfte, die im interdisziplinären Team einen schweren Verlauf einer Covid-19, also ein Lungenversagen, professionell begleiten. Wir müssen sämtliche Versorgungsstrukturen stärken, insbesondere die Altenpflege und die Außerklinik. Schon jetzt warnt die Deutsche interdisziplinäre Gesellschaft für außerklinische Beatmung (DIGAB), dass die Außerklinik von Engpässen mit Beatmungsequipment betroffen sein wird. Corona könnte zum Sprengstoff für sämtliche Versorgungsstrukturen werden.

_ Sie haben gemeinsam mit Kollegen eine Online-Petition an Bundesgesundheitsminister Jens Spahn gestartet. Ist jetzt die richtige Zeit, um Forderungen zu stellen? Falckner: Ja, jetzt ist genau die richtige Zeit, insbesondere auch für die Forderung nach einem Einstiegsgehalt von 4.000 Euro brutto für Pflegefachkräfte. Wir müssen unsere Kolleginnen und Kollegen im Pflexit, also die, die dem als eine Fachkraft im 3-Schichtsystem mit Verantwortung über Leben und Tod.

_ Sie sind Mitinitiatorin des Berliner Care Slams, der Missstände in der Pflege durchaus auch mit Humor aufgreift. Bleibt Ihnen manchmal das Lachen im Hals stecken? Falckner: Pflege bewegt sich sehr häufig schon im Rahmen von Realsatire. Das lässt mich oft traurig zurück. In meiner Familie wurde immer viel gelacht, obwoh/ wir alle ernste Menschen sind. Lachen hilft über das Schwierige. Für diese Gabe bin ich dankbar. Ich für meinen Teil nehme die Pflege wirklich sehr ernst und betrachte sie als schützenswertes Gut. Mein Wunsch wäre, dass sich der Gesundheitsminister Spahn den Ritterschlag der Pflegenden holt. Ich würde ihm dann auch einen ganzen CareSlam widmen.

Das Interview führte Nicoletta Eckardt.

Hier geht's zur Petition: change.org/p/ covid2019-gemeinsamer-pflegefachkräfteaufruf-an-jensspahn 